\title{
Screening rates for colorectal cancer in Canada: a cross-sectional study
}

\author{
Harminder Singh MD MPH, Charles N. Bernstein MD, Jewel N. Samadder MD MSc, Rashid Ahmed PhD
}

\section{Abstract}

Introduction: Implementation of population-based colorectal cancer (CRC) screening programs should reduce disparities in participation in CRC screening. We estimated CRC screening rates in 2012 in Canada and assessed predictors of screening in provinces with and without well-established population-based screening programs.

Methods: We used data from the Canadian Community Health Survey for 2012 to calculate the prevalence of up-to-date CRC screening, defined as fecal occult blood testing (FOBT) within 2 years before the survey or flexible sigmoidoscopy or colonoscopy within 10 years before the survey, or both. Weighted proportions of individuals with up-to-date screening were calculated and logistic regression analysis performed to assess predictors of up-to-date CRC screening, including differences in participation by income level.

Results: The prevalence of up-to-date CRC screening among people 50-74 years of age in 2012 was $55.2 \%$, ranging from $41.3 \%$ in the territories to $67.2 \%$ in the province of Manitoba. The rate for sigmoidoscopy or colonoscopy was $37.2 \%$ (highest in Ontario, at 43.3\%), and for FOBT it was $30.1 \%$ (highest in Manitoba, at 51.7\%). About $41 \%$ of those who had an FOBT also had a sigmoidoscopy or colonoscopy. Individuals in the highest income group were more likely than those in lower-income groups to be up to date with CRC screening, even in provinces with well-established population-based screening programs.

Interpretation: More than half of Canadians were up to date with CRC screening in 2012, but there were large differences among provinces. Differences by income group in provinces with population-based screening programs need particular attention.

olorectal cancer (CRC) continues to be the second most common cause of cancer-related deaths and cancer-related premature death in Canada. ${ }^{1}$ Several randomized controlled trials (RCTs) have shown that screening for CRC by fecal occult blood test (FOBT) or by flexible sigmoidoscopy reduces CRC incidence and mortality. ${ }^{2-4}$ Although several RCTs have been started only recently to determine the efficacy of colonoscopy for CRC screening, results of observational studies and indirect evidence support its use. ., $^{5}$

Over the last 2 decades, several North American guidelines have recommended CRC screening among men and women between the ages of 50 and 75 years. ${ }^{7-11}$ The Canadian Task Force on Preventive Health Care $^{8}$ issued its recommendation in 2001, and in 2002 a Health Canada national committee on CRC screening ${ }^{12}$ recommended that all provinces and territories consider launching population-based CRC screening programs. Between 2007 and 2009, 4 provinces (Nova Scotia, Ontario, Manitoba and Saskatchewan) implemented province-wide programs, followed by Prince Edward Island in 2011. ${ }^{13}$ Since then, most of the remaining provinces have introduced or are planning to implement programs. The provincial screening programs use various versions of the FOBT and involve mass media campaigns to promote CRC screening and are discussed in detail elsewhere. ${ }^{13}$
Before the implementation of provincial programs, rates of participation in CRC screening among Canadians were reported to be low, at $24 \%$ (including only $18 \%$ considered to be up to date) in 2003. ${ }^{14}$ More recent estimates suggest a rate of $41 \%$ in Ontario in $2011 .{ }^{15}$ However, recent estimates in other provinces and in Canada overall are not available. In addition, it is not known whether the implementation of population-based CRC screening programs in some provinces has led to a reduction in disparities in rates ${ }^{16}$ among different groups in those provinces. Determination of predictors of CRC screening in provinces with and without well-established screening programs would provide informa-

Competing interests: Harminder Singh has been a consultant to Medial Cancer Screening Ltd. Charles Bernstein is supported in part by the Bingham Chair in Gastroenterology. He has been a consultant to Abbvie Canada, Forest Canada, Takeda Canada and Pfizer. He has received research grants from Abbvie Canada and unrestricted educational grants from Aptalis Pharmaceuticals, Takeda Canada, Shire Canada and Abbvie Canada. Jewel Samadder has been a consultant to Cook Medical Inc. No competing interests were declared by Rashid Ahmed.

This article has been peer reviewed.

Correspondence to: Harminder Singh, Harminder.singh@umanitoba.ca CMAJ Open 2015. DOI:10.9778/cmajo.20140073 
tion for provinces to develop tailored programs for groups with low participation rates.

We estimated up-to-date CRC screening rates in 2012 in Canada. We also assessed predictors of up-to-date screening in provinces with and without well-established populationbased screening programs, including differences in participation rates by income level.

\section{Methods}

\section{Data source}

We obtained data from the master file of the 2012 Canadian Community Health Survey. The survey is a cross-sectional study that uses a multistage, stratified, cluster sampling design to collect information related to health status, health care utiliza-

\begin{tabular}{|c|c|c|c|c|c|c|c|c|c|c|c|c|c|c|}
\hline \multirow[b]{2}{*}{ Characteristic } & \multicolumn{14}{|c|}{ Jurisdiction; \% of population } \\
\hline & $\mathrm{NL}$ & PEI & NS & NB & QC & ON & $\mathrm{MB}$ & SK & $A B$ & $\mathrm{BC}$ & Territories* & $\begin{array}{c}\text { Provinces with } \\
\text { CRC } \\
\text { screening } \\
\text { programs† }\end{array}$ & $\begin{array}{l}\text { Provinces } \\
\text { without CRC } \\
\text { screening } \\
\text { programs }\end{array}$ & Canada \\
\hline \multicolumn{15}{|l|}{ Age, yr } \\
\hline $50-54$ & 17.7 & 22.9 & 21.2 & 24.7 & 25.8 & 26.7 & 25.9 & 26.9 & 25.1 & 27.6 & 30.3 & 26.3 & 25.8 & 26.0 \\
\hline $55-59$ & 25.8 & 22.4 & 29.0 & 19.9 & 23.6 & 23.5 & 24.7 & 23.1 & 27.6 & 21.8 & 28.9 & 23.9 & 23.8 & 23.8 \\
\hline $60-64$ & 25.2 & 25.6 & 19.7 & 24.1 & 21.1 & 22.3 & 21.2 & 22.7 & 21.7 & 21.7 & 21.7 & 22.1 & 21.7 & 21.9 \\
\hline $65-69$ & 20.8 & 17.9 & 16.7 & 19.4 & 16.5 & 16.2 & 14.9 & 15.4 & 16.5 & 17.4 & 14.0 & 16.1 & 17.0 & 16.6 \\
\hline 70-74 & 10.5 & 11.2 & 13.5 & 11.8 & 13.1 & 11.4 & 13.3 & 11.9 & 9.2 & 11.5 & 5.2 & 11.7 & 11.7 & 11.7 \\
\hline \multicolumn{15}{|l|}{ Sex } \\
\hline Male & 47.6 & 48.5 & 47.5 & 48.0 & 49.5 & 48.7 & 48.0 & 47.5 & 48.2 & 48.8 & 51.0 & 48.5 & 48.9 & 48.7 \\
\hline Female & 52.4 & 51.5 & 52.6 & 52.0 & 50.6 & 51.3 & 52.1 & 52.5 & 51.8 & 51.2 & 49.0 & 51.5 & 51.1 & 51.3 \\
\hline \multicolumn{15}{|l|}{ Income, \$ } \\
\hline$<20000$ & 12.3 & 8.0 & 10.5 & 10.2 & 11.0 & 7.4 & 9.0 & 8.4 & 4.4 & 9.8 & 9.0 & 9.5 & 7.7 & 8.6 \\
\hline $20000-39000$ & 24.7 & 27.3 & 25.7 & 28.0 & 24.5 & 18.2 & 15.6 & 17.3 & 13.8 & 19.8 & 14.3 & 21.4 & 18.5 & 20.0 \\
\hline $40000-59999$ & 19.3 & 17.3 & 16.6 & 21.3 & 19.8 & 20.1 & 19.3 & 16.9 & 19.4 & 20.8 & 12.1 & 20.0 & 19.6 & 19.8 \\
\hline $60000-79999$ & 13.8 & 16.2 & 15.8 & 15.0 & 15.4 & 16.2 & 19.0 & 15.2 & 12.4 & 14.5 & 14.4 & 14.6 & 16.3 & 15.4 \\
\hline$\geq 80000$ & 29.9 & 21.3 & 31.5 & 25.5 & 29.3 & 38.2 & 37.1 & 42.2 & 50.0 & 35.2 & 50.2 & 34.6 & 37.9 & 36.1 \\
\hline Missing data & 0.1 & 0.0 & 0.2 & 0.2 & 0.0 & 0.0 & 0.1 & 0.2 & 0.0 & 0.0 & 0.0 & 0.02 & 0.04 & 0.03 \\
\hline \multicolumn{15}{|l|}{ FOBT $<2 y r$} \\
\hline Yes & 20.4 & 42.3 & 37.7 & 19.7 & 14.5 & 35.8 & 51.7 & 32.5 & 38.1 & 31.3 & 25.8 & 36.9 & 23.9 & 30.1 \\
\hline Missing data & 4.0 & 3.1 & 1.8 & 3.1 & 2.8 & 3.3 & 1.2 & 1.9 & 2.8 & 3.8 & 1.5 & 3.1 & 3.0 & 3.1 \\
\hline \multicolumn{15}{|l|}{$\begin{array}{l}\text { FS or } \\
\text { colonoscopy } \\
<10 \mathrm{yr}\end{array}$} \\
\hline Yes & 34.8 & 35.8 & 28.2 & 35.4 & 33.6 & 43.3 & 33.4 & 35.8 & 36.7 & 31.1 & 26.6 & 41.2 & 33.7 & 37.2 \\
\hline Missing data & 2.9 & 4.0 & 1.3 & 2.7 & 2.0 & 2.6 & 1.1 & 1.6 & 2.7 & 3.1 & 1.2 & 2.5 & 2.4 & 2.4 \\
\hline \multicolumn{15}{|l|}{$\begin{array}{l}\text { Either or both } \\
\text { CRC screening } \\
\text { tests }\end{array}$} \\
\hline Yes & 46.0 & 62.1 & 51.5 & 47.3 & 43.4 & 64.1 & 67.2 & 52.8 & 59.5 & 49.6 & 41.3 & 62.8 & 48.4 & 55.2 \\
\hline Missing data & 3.2 & 4.4 & 1.3 & 3.1 & 2.4 & 3.1 & 1.1 & 1.8 & 3.1 & 3.5 & 1.4 & 2.9 & 2.7 & 2.8 \\
\hline \multicolumn{15}{|l|}{$\begin{array}{l}\text { Mammogram } \\
<2 \mathrm{yr}\end{array}$} \\
\hline Yes & 78.5 & 72.0 & 76.3 & 73.8 & 78.5 & 79.1 & 78.8 & 74.4 & 77.9 & 76.2 & 68.7 & 78.7 & 77.5 & 78.0 \\
\hline \multicolumn{15}{|l|}{$\begin{array}{l}\text { Pap smear } \\
<3 \mathrm{yr}\end{array}$} \\
\hline Yes & 69.3 & 72.6 & 64.8 & 57.4 & 67.7 & 69.1 & 79.4 & 63.8 & 70.0 & 68.7 & 73.1 & 69.2 & 68.0 & 68.6 \\
\hline
\end{tabular}


tion and determinants of health among people 12 years of age and older living in private dwellings in the 115 health regions across all provinces and territories. Individuals living on Indian reserves or Crown land, people in institutions, full-time members of the armed forces and residents of certain remote regions (collectively less than 3\% of the Canadian population) are excluded. Dwellings or households are used as the final sampling unit. The survey design allows researchers to have valid and reliable estimates at the health-region level. Details of the sampling procedure have been documented by Statistics Canada. ${ }^{17}$

For our study, we obtained data for respondents aged 40 and over. All of our analyses were performed with the use of survey weights to account for sampling design, one-dimension post-stratification, and nonresponses at the household and individual level..$^{17}$ There were 61707 respondents, sampled to be representative of 29491030 Canadians. In the 2012 Canadian Community Health Survey, questions on CRC screening were included for all provinces and territories.

\section{Definition of up-to-date screening}

We defined up-to-date CRC screening as any exposure to FOBT in the 2 years before the survey or exposure to flexible sigmoidoscopy or colonoscopy in the 10 years before the survey, or both (Appendix 1, available at www.cmajopen.ca/content /3/2/E149/suppl/DC1). These respective intervals were recommended in recent CRC screening guidelines. ${ }^{10}$ Exposure to any FOBT, flexible sigmoidoscopy or colonoscopy was included in the definition, rather than only those performed as "part of regular check-up," because previous studies have shown that patient history may not be reliable in distinguishing screening from nonscreening examinations. ${ }^{18}$ Moreover, use of these tests for nonscreening indications also serves as screening, and therefore the tests do not need to be repeated for screening.

\section{Predictor variables assessed}

We evaluated socioeconomic factors and factors found to be associated with risk of CRC or CRC screening behaviour in prior studies. ${ }^{19-21}$ The sociodemographic variables assessed included age (in 5-year groups), sex, total household income from all sources, highest education level and marital status. We also assessed the effect of obesity, smoking, diabetes, physical examination in the year before the survey, selfdescribed medical status, restriction of physical activities, upto-date status for cervical cancer and breast cancer screening among women (up-to-date status defined based on guidelines from the Canadian Task Force on Preventive Health Care ${ }^{22,23}$ ), and province of residence. Diabetes was used as a marker for a common chronic disease. We calculated body mass indexes from self-reported height and weight and categorized respondents into different weight classes (underweight, normal, overweight and obese).

\section{Statistical analysis}

We used statistical weights to ensure that the estimates reflected the general Canadian and provincial populations. Weighted proportions are reported. We performed logistic regression analysis to assess predictors of up-to-date CRC screening status. We first conducted logistic regression analyses for the individual potential variables, with adjustment for age and sex a priori. We then developed fully saturated models that included all variables from the age- and sex-adjusted models except education level; provinces were aggregated to those with (Nova Scotia, Ontario, Manitoba and Saskatachewan) and those without well-established population-based CRC screening programs in 2012. Because there is a strong correlation between education level and income, we used only income in the saturated models to avoid multi-collinearity. We used cervical cancer and breast cancer screening in models that included women only. We performed stratified analyses for provinces with and without CRC screening programs and for male and female respondents. Results are reported as odds ratios (ORs) with associated $95 \%$ confidence intervals (CIs).

We restricted the above primary analyses to people within the recommended age group for CRC screening (50-74 yr). We also determined the use of CRC screening tests among younger and older individuals. In addition, we estimated the concomitant use of FOBT in the preceding 2 years and of sigmoidoscopy or colonoscopy in the preceding 10 years. The 2012 Canadian Community Health Survey did not attempt to separate use of sigmoidoscopy and colonoscopy; prior studies suggest that patients' self-reports are relatively poor in separating these 2 tests. $^{24}$

Table 2: Exposure to colorectal cancer screening tests* by age and sex

\begin{tabular}{|c|c|c|c|}
\hline \multirow[b]{2}{*}{ Variable } & \multicolumn{3}{|c|}{ Screening test, $\%$ of patients } \\
\hline & FOBT & $\begin{array}{c}\text { FS or } \\
\text { colonoscopy }\end{array}$ & $\begin{array}{c}\text { Either or } \\
\text { both }\end{array}$ \\
\hline \multicolumn{4}{|c|}{$\begin{array}{l}\text { Within } \\
\text { recommended } \\
\text { age range } \\
(50-74 \text { yr) }\end{array}$} \\
\hline $50-54$ & 21.2 & 27.0 & 41.8 \\
\hline $55-59$ & 29.4 & 36.4 & 54.7 \\
\hline $60-64$ & 34.7 & 40.7 & 61.0 \\
\hline $65-69$ & 34.6 & 43.5 & 62.5 \\
\hline $70-74$ & 36.4 & 46.5 & 65.3 \\
\hline Male & 30.0 & 37.6 & 54.9 \\
\hline Female & 30.2 & 36.9 & 55.5 \\
\hline \multicolumn{4}{|c|}{$\begin{array}{l}\text { Outside } \\
\text { recommended } \\
\text { age range, yr }\end{array}$} \\
\hline $40-44$ & 5.1 & 12.3 & 15.6 \\
\hline $45-49$ & 7.2 & 16.7 & 21.5 \\
\hline $75-79$ & 34.0 & 43.0 & 60.4 \\
\hline$\geq 80$ & 23.4 & 36.8 & 49.7 \\
\hline Male & 12.8 & 20.1 & 27.5 \\
\hline Female & 11.3 & 22.3 & 28.6 \\
\hline
\end{tabular}




\section{OPEN}

\section{Results}

\section{Exposure to $\mathrm{CRC}$ screening tests}

The prevalence of up-to-date self-reported exposure to one or both CRC screening tests in Canada in 2012 was $55.2 \%$ (Table 1). The rate ranged from $41.3 \%$ in the territories (Northwest Territories, Yukon and Nunavut) to $67.2 \%$ in the province of Manitoba. For flexible sigmoidoscopy or colonoscopy, the rate was $37.2 \%$ overall, and for FOBT it was $30.1 \%$. The highest rate of sigmoidoscopy or colonoscopy was in Ontario (43.3\%), and the highest rate of FOBT use was in Manitoba (51.7\%). Overall, the rates of CRC screening were higher in the provinces with well-established CRC screening programs (FOBT: $36.9 \%$, sigmoidoscopy or colonoscopy: $41.2 \%$, either or both tests: $62.8 \%$ ); however, Alberta and Prince Edward Island (provinces without such programs in 2012) had among the highest screening rates (Table 1). There was less interprovincial variation in the rates of exposure to Pap testing and mammography (Table 1), for which screening programs exist across Canada.

The demographic age and sex distribution of individuals 50-74 years old is listed in Table 1 and the respective exposure to CRC screening tests in Table 2. The rates of FOBT and of sigmoidoscopy or colonoscopy increased with age. People aged 70-74 had the highest rate of CRC screening (65.3\%). The CRC screening rate among women $(55.5 \%)$ was markedly lower than that of Pap testing (68.6\%) or mammography (78.0\%) among women 50-74 (Tables 1 and 2). CRC screening rates among individuals outside of the recommended range are also shown in Table 2.

About $41 \%$ of the individuals $50-74$ years old who had an FOBT also had a sigmoidoscopy or colonoscopy. Conversely, one-third of those who had a sigmoidoscopy or colonoscopy also had an FOBT.

The self-reported indications for sigmoidoscopy or colonoscopy are listed in Table 3. Overall, 33.1\% of those 50-74 years of age reported that their tests were performed for follow-up of a medical problem and $55.7 \%$ for screening or because of their age. Because of concerns of the reliability of self-reports of indications, we performed no additional analyses of indications.

\section{Predictors of CRC screening}

In the analysis of predictors of CRC screening among individuals aged 50-74 years, adjustment for only age and sex showed that higher education, higher income and not being a current smoker were associated with increased odds of CRC screening. Being unmarried, being obese, feeling well (i.e., self-described medical status of fair or better and no restrictions of physical

Table 3: Self-reported indications for flexible sigmoidoscopy or colonoscopy within preceding 10 years

\begin{tabular}{|c|c|c|c|c|c|c|c|c|c|c|c|c|}
\hline \multirow[b]{2}{*}{ Variable } & \multicolumn{12}{|c|}{ Jurisdiction; \% of respondents who had test } \\
\hline & NL & PEI & NS & NB & QC & ON & MB & SK & $A B$ & $\mathrm{BC}$ & Territories* & Canada \\
\hline \multicolumn{13}{|l|}{ Age 50-74 yr } \\
\hline Family history & 30.9 & 19.6 & 17.8 & 23.3 & 18.5 & 15.4 & 13.1 & 18.5 & 18.5 & 20.0 & 24.9 & 17.5 \\
\hline Routine screening & 23.8 & 23.6 & 36.2 & 33.5 & 35.0 & 55.6 & 36.1 & 36.7 & 43.5 & 36.3 & 41.6 & 45.0 \\
\hline Age & 2.2 & 9.8 & 4.9 & 8.8 & 6.3 & 15.6 & 8.6 & 7.9 & 8.6 & 5.8 & 9.5 & 10.7 \\
\hline Follow-up of problem & 45.9 & 56.4 & 46.9 & 43.1 & 35.6 & 28.5 & 38.3 & 37.7 & 30.7 & 38.7 & 28.9 & 33.1 \\
\hline $\begin{array}{l}\text { Follow-up of CRC } \\
\text { treatment }\end{array}$ & 3.3 & 0.1 & 3.1 & 2.9 & 2.9 & 1.3 & 1.6 & 3.3 & 1.6 & 1.8 & 2.6 & 1.9 \\
\hline \multicolumn{13}{|l|}{ Age 40-49 yr } \\
\hline Family history & 48.3 & 13.4 & 18.4 & 44.0 & 17.0 & 28.4 & 32.3 & 32.8 & 23.9 & 28.6 & 30.6 & 25.8 \\
\hline Routine screening & 17.1 & 0.0 & 14.5 & 11.3 & 14.0 & 27.5 & 14.2 & 4.5 & 10.2 & 12.6 & 19.5 & 18.4 \\
\hline Age & 0.0 & 0.0 & 0.0 & 1.2 & 5.2 & 3.0 & 3.7 & 0.9 & 0.7 & 0.6 & $1 . .2$ & 2.8 \\
\hline Follow-up of problem & 35.6 & 86.6 & 72.5 & 47.3 & 56.6 & 48.2 & 50.9 & 62.8 & 47.4 & 64.1 & 50.0 & 52.6 \\
\hline $\begin{array}{l}\text { Follow-up of CRC } \\
\text { treatment }\end{array}$ & 1.2 & 0.0 & 0.0 & 0.4 & 0.1 & 0 & 0 & 0 & 0 & 0.5 & 0.8 & 0.2 \\
\hline \multicolumn{13}{|l|}{ Age $\geq 74$ yr } \\
\hline Family history & 11.1 & 12.5 & 12.1 & 15.1 & 9.5 & 7.7 & 10.4 & 16.2 & 8.6 & 10.4 & 12.1 & 9.3 \\
\hline Routine screening & 21.2 & 30.7 & 30.8 & 34.3 & 45.3 & 57.7 & 38.9 & 33.5 & 50.0 & 43.8 & 53.4 & 49.2 \\
\hline Age & 0.0 & 2.1 & 1.6 & 3.7 & 5.5 & 11.8 & 1.0 & 1.8 & 11.8 & 3.4 & 0.0 & 7.9 \\
\hline Follow-up of problem & 65.8 & 56.2 & 49.2 & 50.8 & 34.8 & 33.5 & 44.9 & 51.4 & 29.7 & 38.7 & 28.9 & 36.6 \\
\hline $\begin{array}{l}\text { Follow-up of CRC } \\
\text { treatment }\end{array}$ & 4.9 & 0.0 & 8.3 & 4.0 & 5.7 & 3.7 & 3.1 & 3.7 & 2.7 & 3.4 & 5.6 & 4.2 \\
\hline
\end{tabular}


Table 4: Predictors of up-to-date CRC screening, stratified by provinces with and without CRC screening programs in 2012 (age- and sex-adjusted logistic regression analysis)

\begin{tabular}{|c|c|c|c|c|}
\hline \multirow[b]{2}{*}{ Variable } & \multirow[b]{2}{*}{$\%$ in Canada } & \multicolumn{3}{|c|}{ OR $(95 \% \mathrm{Cl})$} \\
\hline & & All provinces & $\begin{array}{l}\text { Provinces with } \\
\text { screening programs* }\end{array}$ & $\begin{array}{l}\text { Provinces without } \\
\text { screening programs }\end{array}$ \\
\hline \multicolumn{5}{|l|}{ Education } \\
\hline$<$ Secondary school & 17.2 & 1.00 (ref) & 1.00 (ref) & 1.00 (ref) \\
\hline $\begin{array}{l}\text { Secondary school } \\
\text { graduation }\end{array}$ & 18.7 & $1.38(1.27-1.51)$ & $1.43(1.26-1.63)$ & $1.27(1.13-1.44)$ \\
\hline Some postsecondary school & 4.2 & $1.31(1.14-1.51)$ & $1.79(1.41-2.27)$ & $1.13(0.94-1.35)$ \\
\hline $\begin{array}{l}\text { Postsecondary certification/ } \\
\text { diploma or university }\end{array}$ & 59.9 & $1.50(1.40-1.61)$ & $1.62(1.45-1.81)$ & $1.38(1.25-1.52)$ \\
\hline \multicolumn{5}{|l|}{ Income, \$ } \\
\hline$<20000$ & 8.6 & 1.00 (ref) & 1.00 (ref) & 1.00 (ref) \\
\hline $20000-39000$ & 20.0 & $1.08(0.98-1.20)$ & $1.10(0.94-1.29)$ & $1.07(0.93-1.22)$ \\
\hline $40000-59999$ & 19.8 & $1.31(1.18-1.45)$ & $1.30(1.10-1.52)$ & $1.28(1.11-1.47)$ \\
\hline $60000-79999$ & 15.4 & $1.37(1.23-1.52)$ & $1.44(1.22-1.70)$ & $1.22(1.06-1.41)$ \\
\hline$\geq 80000$ & 36.1 & $1.85(1.68-2.04)$ & $1.97(1.69-2.29)$ & $1.65(1.45-1.88)$ \\
\hline \multicolumn{5}{|l|}{ Marital status } \\
\hline Married & 65.4 & 1.00 (ref) & 1.00 (ref) & 1.00 (ref) \\
\hline Common law & 8.1 & $0.76(0.69-0.84)$ & $1.09(0.92-1.28)$ & $0.73(0.65-0.82)$ \\
\hline Widowed/separated/divorced & 18.5 & $0.71(0.66-0.76)$ & $0.71(0.64-0.78)$ & $0.72(0.66-0.79)$ \\
\hline Single (never married) & 8.0 & $0.63(0.58-0.70)$ & $0.78(0.67-0.91)$ & $0.60(0.53-0.69)$ \\
\hline \multicolumn{5}{|l|}{ Obesity } \\
\hline Underweight/normal & 39.0 & 1.00 (ref) & 1.00 (ref) & 1.00 (ref) \\
\hline Overweight & 38.8 & $1.02(0.95-1.10)$ & $1.00(0.90-1.11)$ & $1.05(0.96-1.15)$ \\
\hline Obese & 22.2 & $0.80(0.75-0.86)$ & $0.77(0.69-0.85)$ & $0.85(0.78-0.93)$ \\
\hline \multicolumn{5}{|l|}{ Smoking status } \\
\hline Daily & 18.7 & 1.00 (ref) & 1.00 (ref) & 1.00 (ref) \\
\hline Occasional/former & 50.0 & $1.95(1.82-2.09)$ & $2.05(1.85-2.28)$ & $2.00(1.82-2.20)$ \\
\hline Never smoked & 31.3 & $1.67(1.55-1.80)$ & $1.54(1.38-1.71)$ & $1.73(1.55-1.92)$ \\
\hline \multicolumn{5}{|l|}{ Diabetes } \\
\hline No & 87.9 & 1.00 (ref) & 1.00 (ref) & 1.00 (ref) \\
\hline Yes & 12.1 & $0.98(0.91-1.06)$ & $0.83(0.74-0.94)$ & $1.10(0.98-1.22)$ \\
\hline \multicolumn{5}{|l|}{ Physical examination } \\
\hline No regular pattern & 43.1 & 1.00 (ref) & 1.00 (ref) & 1.00 (ref) \\
\hline At least once annually & 56.9 & $0.47(0.44-0.50)$ & $0.43(0.40-0.47)$ & $0.49(0.46-0.53)$ \\
\hline \multicolumn{5}{|l|}{$\begin{array}{l}\text { Self-described general } \\
\text { medical status }\end{array}$} \\
\hline Poor & 4.0 & 1.00 (ref) & 1.00 (ref) & 1.00 (ref) \\
\hline Fair & 10.6 & $0.81(0.70-0.95)$ & $0.93(0.75-1.16)$ & $0.78(0.62-0.97)$ \\
\hline Good & 32.1 & $0.85(0.73-0.98)$ & $1.02(0.83-1.24)$ & $0.77(0.63-0.95)$ \\
\hline Very good & 35.8 & $0.87(0.76-1.00)$ & $1.16(0.95-1.41)$ & $0.71(0.58-0.88)$ \\
\hline Excellent & 17.4 & $0.84(0.73-0.98)$ & $1.12(0.91-1.38)$ & $0.70(0.56-0.87)$ \\
\hline \multicolumn{5}{|l|}{ Restriction of activities } \\
\hline Sometimes & 19.6 & 1.00 (ref) & 1.00 (ref) & 1.00 (ref) \\
\hline Often & 14.6 & $0.95(0.87-1.04)$ & $0.97(0.85-1.11)$ & $0.91(0.81-1.03)$ \\
\hline Never & 65.8 & $0.84(0.79-0.90)$ & $0.99(0.89-1.09)$ & $0.71(0.65-0.78)$ \\
\hline \multicolumn{5}{|l|}{ Pap test } \\
\hline None or $\geq 3 \mathrm{yr}$ & 31.4 & 1.00 (ref) & 1.00 (ref) & 1.00 (ref) \\
\hline$<3 \mathrm{yr}$ & 68.6 & $1.86(1.71-2.03)$ & $2.29(2.03-2.60)$ & $1.58(1.41-1.78)$ \\
\hline \multicolumn{5}{|l|}{ Mammogram } \\
\hline None or $\geq 2 \mathrm{yr}$ & 22.0 & 1.00 (ref) & 1.00 (ref) & 1.00 (ref) \\
\hline$<2 \mathrm{yr}$ & 78.0 & $2.58(2.35-2.82)$ & $3.61(3.15-4.13)$ & $2.02(1.78-2.29)$ \\
\hline \multicolumn{5}{|l|}{ Province } \\
\hline Manitoba & 3.3 & 1.00 (ref) & 1.00 (ref)‡ & - \\
\hline Newfoundland and Labrador & 1.7 & $0.38(0.30-0.48)$ & - & $0.50(0.33-0.75)$ \\
\hline Prince Edward Island & 0.5 & $0.78(0.52-1.16)$ & - & 1.00 (ref)‡ \\
\hline Nova Scotia & 3.2 & $0.49(0.40-0.60)$ & $0.48(0.39-0.59)$ & - \\
\hline New Brunswick & 2.6 & $0.42(0.34-0.52)$ & - & $0.54(0.36-0.80)$ \\
\hline Quebec & 24.4 & $0.36(0.31-0.42)$ & - & $0.46(0.32-0.67)$ \\
\hline Ontario & 38.3 & $0.87(0.75-1.02)$ & $0.87(0.75-1.02)$ & - \\
\hline Saskatchewan & 2.7 & $0.54(0.44-0.67)$ & $0.53(0.43-0.66)$ & - \\
\hline Alberta & 9.7 & $0.72(0.61-0.85)$ & - & $0.92(0.63-1.34)$ \\
\hline British Columbia & 13.5 & $0.47(0.40-0.55)$ & - & $0.61(0.42-0.88)$ \\
\hline Territories† & 0.2 & $0.36(0.20-0.63)$ & - & $0.45(0.23-0.88)$ \\
\hline \multicolumn{5}{|c|}{$\begin{array}{l}\text { Note: } \mathrm{Cl}=\text { confidence interval, } \mathrm{CRC}=\text { colorectal cancer, ref = reference group. } \\
\text { *Nova Scotia, Ontario, Manitoba and Saskatchewan. } \\
\text { †Northwest Territories, Yukon and Nunavut. } \\
\text { fProvinces with the highest rates of screening in the respective groups (Manitoba for those with screening programs and PEI for those } \\
\text { without screening programs) were used as the reference groups. }\end{array}$} \\
\hline
\end{tabular}




\section{OPEN}

\begin{tabular}{|c|c|c|c|}
\hline \multirow[b]{2}{*}{ Variable } & \multicolumn{3}{|c|}{ OR $(95 \% \mathrm{Cl})$} \\
\hline & All provinces & $\begin{array}{c}\text { Provinces with } \\
\text { screening programs* }\end{array}$ & $\begin{array}{l}\text { Provinces without } \\
\text { screening programs }\end{array}$ \\
\hline \multicolumn{4}{|l|}{ Age, yr } \\
\hline $50-54$ & 1.00 (ref) & 1.00 (ref) & 1.00 (ref) \\
\hline $55-59$ & $1.70(1.57-1.84)$ & $1.78(1.59-2.00)$ & $1.64(1.47-1.83)$ \\
\hline $60-64$ & $2.21(2.03-2.40)$ & $2.67(2.35-3.03)$ & $1.92(1.72-2.16)$ \\
\hline $65-69$ & $2.33(2.12-2.56)$ & $2.97(2.57-3.43)$ & $1.96(1.72-2.22)$ \\
\hline $70-74$ & $2.67(2.40-2.98)$ & $3.08(2.61-3.64)$ & $2.37(2.05-2.74)$ \\
\hline \multicolumn{4}{|l|}{ Sex } \\
\hline Female & 1.00 (ref) & 1.00 (ref) & 1.00 (ref) \\
\hline Male & $1.02(0.96-1.09)$ & $0.91(0.83-0.99)$ & $1.12(1.04-1.22)$ \\
\hline \multicolumn{4}{|l|}{ Income, \$ } \\
\hline$<20000$ & 1.00 (ref) & 1.00 (ref) & 1.00 (ref) \\
\hline $20000-39000$ & $1.01(0.90-1.15)$ & $1.07(0.89-1.30)$ & $0.99(0.84-1.16)$ \\
\hline $40000-59999$ & $1.17(1.04-1.33)$ & $1.25(1.03-1.52)$ & $1.15(0.98-1.36)$ \\
\hline $60000-79999$ & $1.11(0.98-1.27)$ & $1.33(1.09-1.62)$ & $1.01(0.85-1.20)$ \\
\hline$\geq 80000$ & $1.50(1.33-1.70)$ & $1.75(1.45-2.12)$ & $1.38(1.17-1.62)$ \\
\hline \multicolumn{4}{|l|}{ Marital status } \\
\hline Married & 1.00 (ref) & 1.00 (ref) & 1.00 (ref) \\
\hline Common-law & $0.84(0.76-0.94)$ & $1.16(0.97-1.40)$ & $0.72(0.64-0.82)$ \\
\hline Widowed/separated/divorced & $0.90(0.83-0.98)$ & $0.96(0.85-1.09)$ & $0.86(0.77-0.96)$ \\
\hline Single (never married) & $0.94(0.84-1.06)$ & $1.23(1.02-1.48)$ & $0.80(0.69-0.92)$ \\
\hline \multicolumn{4}{|l|}{ Obesity } \\
\hline Underweight/normal & 1.00 (ref) & 1.00 (ref) & 1.00 (ref) \\
\hline Overweight & $1.08(1.00-1.17)$ & $0.98(0.87-1.10)$ & $1.19(1.07-1.33)$ \\
\hline Obese & $0.89(0.82-0.96)$ & $0.83(0.74-0.93)$ & $0.94(0.85-1.05)$ \\
\hline \multicolumn{4}{|l|}{ Smoking } \\
\hline Daily & 1.00 (ref) & 1.00 (ref) & 1.00 (ref) \\
\hline Occasional/former & $1.81(1.67-1.96)$ & $1.78(1.58-2.01)$ & $1.83(1.64-2.03)$ \\
\hline Never smoked & $1.55(1.42-1.69)$ & $1.42(1.25-1.61)$ & $1.66(1.47-1.88)$ \\
\hline \multicolumn{4}{|l|}{ Diabetes } \\
\hline No & 1.00 (ref) & 1.00 (ref) & 1.00 (ref) \\
\hline Yes & $0.91(0.83-1.01)$ & $0.79(0.69-0.91)$ & $1.01(0.89-1.15)$ \\
\hline \multicolumn{4}{|l|}{ Physical examination } \\
\hline No regular pattern & 1.00 (ref) & 1.00 (ref) & 1.00 (ref) \\
\hline At least once annually & $0.47(0.44-0.50)$ & $0.41(0.38,0.45)$ & $0.51(0.47,0.55)$ \\
\hline \multicolumn{4}{|c|}{$\begin{array}{l}\text { Self-described general medical } \\
\text { status }\end{array}$} \\
\hline Poor & 1.00 (ref) & 1.00 (ref) & 1.00 (ref) \\
\hline Fair & $0.78(0.65-0.94)$ & $0.76(0.58-0.99)$ & $0.74(0.57-0.97)$ \\
\hline Good & $0.74(0.62-0.89)$ & $0.73(0.57-0.94)$ & $0.73(0.56-0.93)$ \\
\hline Very good & $0.72(0.60-0.86)$ & $0.79(0.61-1.02)$ & $0.64(0.49-0.82)$ \\
\hline Excellent & $0.75(0.62-0.90)$ & $0.74(0.56-0.98)$ & $0.71(0.54-0.92)$ \\
\hline \multicolumn{4}{|l|}{ Restriction of activities } \\
\hline Sometimes & 1.00 (ref) & 1.00 (ref) & 1.00 (ref) \\
\hline Often & $1.05(0.94-1.16)$ & $1.22(1.04-1.43)$ & $0.94(0.82-1.08)$ \\
\hline Never & $0.83(0.77-0.90)$ & $1.04(0.93-1.17)$ & $0.70(0.63-0.78)$ \\
\hline \multicolumn{4}{|l|}{ Province } \\
\hline With screening programs & $1.91(1.80-2.02)$ & - & - \\
\hline Without screening programs & 1.00 (ref) & - & - \\
\hline
\end{tabular}


Table 6: Predictors of up-to-date CRC screening in Canada in 2012, stratified by sex and by provinces with and without CRC screening programs in 2012

\begin{tabular}{|c|c|c|c|c|c|c|}
\hline \multirow[b]{2}{*}{ Variable } & \multicolumn{3}{|c|}{ Women; OR (95\% Cl) } & \multicolumn{3}{|c|}{ Men; OR (95\% Cl) } \\
\hline & All provinces & $\begin{array}{c}\text { Provinces } \\
\text { with screening } \\
\text { programs* }\end{array}$ & $\begin{array}{c}\text { Provinces } \\
\text { without screening } \\
\text { programs }\end{array}$ & All provinces & $\begin{array}{c}\text { Provinces } \\
\text { with screening } \\
\text { programs }{ }^{\star}\end{array}$ & $\begin{array}{c}\text { Provinces } \\
\text { without screening } \\
\text { programs }\end{array}$ \\
\hline \multicolumn{7}{|l|}{ Age, yr } \\
\hline $50-54$ & 1.00 (ref) & 1.00 (ref) & 1.00 (ref) & 1.00 (ref) & 1.00 (ref) & 1.00 (ref) \\
\hline $55-59$ & $1.90(1.68-2.16)$ & $1.89(1.56-2.28)$ & $1.87(1.58-2.23)$ & $1.65(1.47-1.85)$ & $1.62(1.37-1.92)$ & $1.71(1.46-2.00)$ \\
\hline $60-64$ & $2.11(1.85-2.42)$ & $2.20(1.79-2.69)$ & $2.08(1.73-2.49)$ & $2.38(2.11-2.69)$ & $2.86(2.37-3.44)$ & $2.07(1.75-2.44)$ \\
\hline $65-69$ & $2.66(2.29-3.08)$ & $2.81(2.24-3.52)$ & $2.53(2.07-3.08)$ & $2.09(1.82-2.40)$ & $2.82(2.27-3.50)$ & $1.72(1.43-2.07)$ \\
\hline $70-74$ & $3.45(2.89-4.11)$ & $3.87(2.95-5.07)$ & $3.10(2.46-3.92)$ & $2.76(2.35-3.24)$ & $3.29(2.56-4.21)$ & $2.46(1.98-3.05)$ \\
\hline \multicolumn{7}{|l|}{ Income, \$ } \\
\hline$<20000$ & 1.00 (ref) & 1.00 (ref) & 1.00 (ref) & 1.00 (ref) & 1.00 (ref) & 1.00 (ref) \\
\hline $20000-39000$ & $1.04(0.86-1.24)$ & $1.01(0.76-1.34)$ & $1.07(0.84-1.36)$ & $0.79(0.64-0.97)$ & $1.10(0.80-1.51)$ & $0.65(0.50-0.84)$ \\
\hline $40000-59999$ & $1.12(0.93-1.35)$ & $1.17(0.88-1.57)$ & $1.12(0.88-1.44)$ & $0.96(0.79-1.18)$ & $1.18(0.85-1.62)$ & $0.90(0.68-1.17)$ \\
\hline $60000-79999$ & $1.07(0.88-1.31)$ & $0.98(0.73-1.33)$ & $1.17(0.89-1.52)$ & $0.95(0.78-1.17)$ & $1.60(1.16-2.21)$ & $0.68(0.51-0.89)$ \\
\hline$\geq 80000$ & $1.61(1.34-1.94)$ & $1.46(1.10-1.95)$ & $1.71(1.33-2.19)$ & $1.17(0.96-1.42)$ & $1.74(1.27-2.37)$ & $0.92(0.71-1.19)$ \\
\hline \multicolumn{7}{|l|}{ Marital status } \\
\hline Married & 1.00 (ref) & 1.00 (ref) & 1.00 (ref) & 1.00 (ref) & 1.00 (ref) & 1.00 (ref) \\
\hline Common-law & $0.87(0.73-1.03)$ & $1.53(1.08-2.16)$ & $0.69(0.56-0.85)$ & $0.78(0.68-0.90)$ & $1.02(0.80-1.31)$ & $0.69(0.58-0.83)$ \\
\hline $\begin{array}{l}\text { Widowed/separated/ } \\
\text { divorced }\end{array}$ & $1.05(0.93-1.18)$ & $0.99(0.83-1.17)$ & $1.09(0.93-1.28)$ & $0.78(0.69-0.89)$ & $0.91(0.75-1.11)$ & $0.69(0.58-0.82)$ \\
\hline Single (never married) & $1.22(1.01-1.47)$ & $1.52(1.11-2.08)$ & $1.06(0.84-1.34)$ & $0.86(0.73-1.02)$ & $1.07(0.81-1.42)$ & $0.74(0.60-0.92)$ \\
\hline \multicolumn{7}{|l|}{ Obesity } \\
\hline Underweight/normal & 1.00 (ref) & 1.00 (ref) & 1.00 (ref) & 1.00 (ref) & 1.00 (ref) & 1.00 (ref) \\
\hline Overweight & $0.86(0.75-0.97)$ & $0.54(0.45-0.66)$ & $1.28(1.08-1.53)$ & $1.26(1.13-1.40)$ & $1.43(1.22-1.67)$ & $1.10(0.94-1.27)$ \\
\hline Obese & $0.82(0.73-0.93)$ & $0.62(0.51-0.75)$ & $1.06(0.89-1.25)$ & $0.91(0.81-1.02)$ & $0.95(0.80-1.13)$ & $0.85(0.72-0.99)$ \\
\hline \multicolumn{7}{|l|}{ Smoking } \\
\hline Daily & 1.00 (ref) & 1.00 (ref) & 1.00 (ref) & 1.00 (ref) & 1.00 (ref) & 1.00 (ref) \\
\hline Occasional/former & $1.68(1.47-1.91)$ & $1.56(1.27-1.90)$ & $1.78(1.49-2.12)$ & $1.82(1.63-2.03)$ & $1.81(1.53-2.13)$ & $1.82(1.56-2.12)$ \\
\hline Never smoked & $1.39(1.22-1.59)$ & $1.25(1.02-1.52)$ & $1.56(1.29-1.88)$ & $1.71(1.50-1.95)$ & $1.61(1.33-1.94)$ & $1.86(1.55-2.23)$ \\
\hline \multicolumn{7}{|l|}{ Diabetes } \\
\hline No & 1.00 (ref) & 1.00 (ref) & 1.00 (ref) & 1.00 (ref) & 1.00 (ref) & 1.00 (ref) \\
\hline Yes & $0.75(0.64-0.88)$ & $0.69(0.55-0.86)$ & $0.78(0.62-0.98)$ & $1.02(0.89-1.16)$ & $0.79(0.65-0.97)$ & $1.22(1.02-1.45)$ \\
\hline \multicolumn{7}{|l|}{ Physical examination } \\
\hline No regular pattern & 1.00 (ref) & 1.00 (ref) & 1.00 (ref) & 1.00 (ref) & 1.00 (ref) & 1.00 (ref) \\
\hline At least once annually & $0.60(0.55-0.66)$ & $0.56(0.48-0.65)$ & $0.62(0.55-0.70)$ & $0.44(0.41-0.48)$ & $0.43(0.38-0.48)$ & $0.45(0.40-0.50)$ \\
\hline \multicolumn{7}{|l|}{$\begin{array}{l}\text { Self-described general } \\
\text { medical status }\end{array}$} \\
\hline Poor & 1.00 (ref) & 1.00 (ref) & 1.00 (ref) & 1.00 (ref) & 1.00 (ref) & 1.00 (ref) \\
\hline Fair & $0.55(0.40-0.74)$ & $0.90(0.59-1.40)$ & $0.29(0.18-0.45)$ & $1.10(0.84-1.43)$ & $0.79(0.54-1.18)$ & $1.41(0.97-2.05)$ \\
\hline Good & $0.52(0.39-0.70)$ & $0.85(0.56-1.27)$ & $0.29(0.18-0.44)$ & $1.07(0.84-1.37)$ & $0.65(0.45-0.94)$ & $1.56(1.11-2.20)$ \\
\hline Very good & $0.51(0.38-0.68)$ & $0.81(0.53-1.23)$ & $0.28(0.18-0.43)$ & $0.94(0.73-1.21)$ & $0.71(0.49-1.04)$ & $1.13(0.80-1.60)$ \\
\hline Excellent & $0.53(0.39-0.72)$ & $0.99(0.64-1.54)$ & $0.26(0.16-0.41)$ & $1.10(0.85-1.44)$ & $0.62(0.42-0.92)$ & $1.65(1.15-2.38)$ \\
\hline \multicolumn{7}{|l|}{ Restriction of activities } \\
\hline Sometimes & 1.00 (ref) & 1.00 (ref) & 1.00 (ref) & 1.00 (ref) & 1.00 (ref) & 1.00 (ref) \\
\hline Often & $0.93(0.80-1.10)$ & $1.16(0.91-1.47)$ & $0.82(0.66-1.01)$ & $1.28(1.09-1.50)$ & $1.68(1.32-2.15)$ & $1.04(0.85-1.29)$ \\
\hline Never & $0.86(0.77-0.97)$ & $1.03(0.87-1.23)$ & $0.73(0.63-0.86)$ & $0.83(0.74-0.92)$ & $1.11(0.94-1.31)$ & $0.66(0.57-0.77)$ \\
\hline \multicolumn{7}{|l|}{ Pap test } \\
\hline None or $\geq 3 \mathrm{yr}$ & 1.00 (ref) & 1.00 (ref) & 1.00 (ref) & - & - & - \\
\hline$<3 \mathrm{yr}$ & $1.24(1.11-1.37)$ & $1.35(1.15-1.58)$ & $1.15(1.00-1.32)$ & - & - & - \\
\hline \multicolumn{7}{|l|}{ Mammmogram } \\
\hline None or $\geq 2 \mathrm{yr}$ & 1.00 (ref) & 1.00 (ref) & 1.00 (ref) & - & - & - \\
\hline$<2 \mathrm{yr}$ & $2.16(1.93-2.42)$ & $2.86(2.42-3.37)$ & $1.61(1.38-1.88)$ & - & - & - \\
\hline \multicolumn{7}{|l|}{ Province } \\
\hline With screening programs & 1.00 (ref) & - & - & 1.00 (ref) & - & - \\
\hline $\begin{array}{l}\text { Without screening } \\
\text { programs }\end{array}$ & $2.30(2.10-2.52)$ & - & - & $1.74(1.60-1.89)$ & - & - \\
\hline
\end{tabular}


activities) and having a physical examination at least once annually were associated with decreased odds of screening (Table 4).

In the fully saturated model, increasing age, annual income of at least $\$ 80000$ and not being a current smoker were associated with increased exposure to CRC screening (Table 5). Being widowed/separated/divorced or common-law, feeling well and having a physical examination at least once a year were associated with decreased odds of screening. Having diabetes was not a predictor of CRC screening. In the fully saturated model stratified by the presence or absence of CRC screening programs (Table 5), the variations in CRC screening by age and income were greater in provinces with programs than in provinces without programs.

In the analysis stratified by sex (Table 6), the results were similar to those of the nonstratified analysis. Up-to-date status with Pap testing and mammography was associated with increased CRC screening among women.

\section{Interpretation}

Our assessment of the prevalence and predictors of CRC screening in 2012 suggests several key findings. First, CRC screening rates have increased in Canada, with over half the population in 2012 being up to date with CRC screening and some provinces having rates similar to those in the United States (which has among the highest CRC screening rates in the world). ${ }^{25-29}$ However, we found large differences between the provinces. For example, an absolute difference in rates of about 25\% existed between Quebec and Manitoba. Second, the test modality varied between the provinces, with higher use of flexible sigmoidoscopy or colonoscopy in Ontario and Quebec, and higher use of FOBT in Manitoba. Third, both the provinces with and those without well-established CRC screening programs had gradients or disparities between income (or education) levels in the use of CRC screening tests. Fourth, within the recommended age range of 50-74 years, CRC screening rates were lowest among individuals $50-55$ years old. Fifth, a substantial minority of individuals outside the recommended age range had CRC screening tests, and as many as $41 \%$ of those who had FOBT also had sigmoidoscopy or colonoscopy. Sixth, obese individuals, daily smokers and those who felt well (i.e., described their medical status as fair or better and had no restrictions of physical activities) had decreased odds of CRC screening. These individuals should be target groups for CRC screening programs.

One of the proposed strengths of population-based CRC screening programs is wider and more equitable coverage of the population. Our results suggest that such programs have not yet narrowed the socioeconomic disparities in CRC screening. Increasing income disparities in CRC screening rates were reported in a recent analysis of health care administrative data in Ontario ${ }^{15}$ however, health care administrative data may not capture FOBTs performed in block-funded facilities. Therefore, our study provides complimentary evidence and includes Canada-wide data. The income disparities in CRC screening rates are concerning, especially because we recently reported widening disparities in CRC mortality by income group. ${ }^{30}$ The results of these 2 studies suggest that people who need higher rates of CRC screening because they have higher CRC-related mortality are less likely to receive CRC screening and therefore should be a target group for CRC screening programs.

Similar to results from other studies, ${ }^{25,29}$ we found lower rates of CRC screening among people 50-55 years old. However, this group may not be an appropriate age group to target, because it has lower CRC incidence and mortality than older groups and stratified analysis of trials of FOBT have not shown benefits of CRC screening in the 50-55 age group..$^{31,32}$ Screening of healthy individuals above the age of 75 , especially those with no prior CRC screening, may be appropriate, because they have higher CRC incidence and mortality than younger individuals $;{ }^{1}$ no CRC screening RCT has included people older than 75. Although the decreased odds of CRC screening among single men was not statistically significant in our study, the trend we observed supports findings in other studies. ${ }^{19}$

\section{Limitations}

The limitations of our study include recall bias, as is inherent in survey studies. The CRC screening rates in different age bands reflect screening experienced when the respondents were younger. However, the differences in FOBT use by age reflect experience in the immediate 2 years before the survey. Because the Canadian Community Health Survey excludes people living on Indian reserves, those in long-term care and members of the armed forces, the results cannot be generalized to those populations. In addition, we were not able to exclude individuals with disorders such as inflammatory bowel disease and hereditary cancer syndromes and those with a high-risk family history of CRC, who require endoscopy on a more regular basis. However, the prevalence of inflammatory bowel disease and hereditary cancer syndromes in the population is less than $1 \% .^{33}$

\section{Conclusion}

Our study showed that more than half of Canadians in the recommended age group of 50-74 years were up to date with CRC screening in 2012. However, we also observed differences in interprovincial screening rates and disparities among income groups, even in provinces with CRC screening programs in place. Screening programs need to be designed to appeal to patients of all socioeconomic levels. The effectiveness of FOBT versus flexible sigmoidoscopy or colonoscopy warrants further investigation.

\section{References}

1. Steering Committee. Canadian cancer statistics 2012. Toronto: Canadian Cancer Society; 2012.

2. Hewitson P, Glasziou P, Watson E, et al. Cochrane systematic review of colorectal cancer screening using the fecal occult blood test (hemoccult): an update. Am 7 Gastroenterol 2008;103:1541-9.

3. Atkin WS, Edwards R, Kralj-Hans I, et al. Once-only flexible sigmoidoscopy screening in prevention of colorectal cancer: a multicentre randomised controlled trial. Lancet 2010;375:1624-33.

4. Schoen RE, Pinsky PF, Weissfeld JL, et al. Colorectal-cancer incidence and mortality with screening flexible sigmoidoscopy. N Engl 7 Med 2012;366: 2345-57. 
5. Brenner H, Stock C, Hoffmeister M. Effect of screening sigmoidoscopy and screening colonoscopy on colorectal cancer incidence and mortality: systematic review and meta-analysis of randomised controlled trials and observational studies. BM7 2014;348:g2467.

6. Thosani N, Guha S, Singh H. Colonoscopy and colorectal cancer incidence and mortality. Gastroenterol Clin North Am 2013;42:619-37.

7. Winawer SJ, Fletcher RH, Miller L, et al. Colorectal cancer screening: clinical guidelines and rationale. Gastroenterology 1997;112:594-642.

8. Canadian Task Force on Preventive Health Care. Colorectal cancer screening. Recommendation statement from the Canadian Task Force on Preventive Health Care. CMA7 2001;165:206-8.

9. Leddin D, Hunt R, Champion M, et al. Canadian Association of Gastroenterology and the Canadian Digestive Health Foundation: Guidelines on colon cancer screening. Can 7 Gastroenterol 2004;18:93-9.

10. Leddin DJ, Enns R, Hilsden R, et al. Canadian Association of Gastroenterology position statement on screening individuals at average risk for developing colorectal cancer: 2010. Can 7 Gastroenterol 2010;24:705-14.

11. US Preventive Services Task Force. Screening for colorectal cancer: US Preventive Services Task Force recommendation statement. Ann Intern Med 2008;149:627-37.

12. Technical report for the National Committee on Colorectal Cancer Screening. Ottawa: Health Canada; 2002.

13. Major D, Bryant H, Delaney M, et al. Colorectal cancer screening in Canada: results from the first round of screening for five provincial programs. Curr Oncol 2013;20:252-7.

14. Zarychanski R, Chen $\mathrm{Y}$, Bernstein $\mathrm{CN}$, et al. Frequency of colorectal cancer screening and the impact of family physicians on screening behaviour. CMAF 2007;177:593-7.

15. Honein-AbouHaidar GN, Baxter NN, Moineddin R, et al. Trends and inequities in colorectal cancer screening participation in Ontario, Canada, 20052011. Cancer Epidemiol 2013;37:946-56.

16. Singh SM, Paszat LF, Li C, et al. Association of socioeconomic status and receipt of colorectal cancer investigations: a population-based retrospective cohort study. CMA7 2004:171:461-5.

17. Béland Y. Canadian Community Health Survey - methodological overview. Health Rep 2002;13:9-14.

18. Schenck AP, Klabunde CN, Warren JL, et al. Data sources for measuring colorectal endoscopy use among Medicare enrollees. Cancer Epidemiol Biomarkers Prev 2007;16:2118-27.

19. Beydoun HA, Beydoun MA. Predictors of colorectal cancer screening behaviors among average-risk older adults in the United States. Cancer Causes Control 2008; 19:339-59.

20. Attner B, Landin-Olsson M, Lithman T, et al. Cancer among patients with diabetes, obesity and abnormal blood lipids: a population-based register study in Sweden. Cancer Causes Control 2012;23:769-77.

21. Liang PS, Chen TY, Giovannucci E. Cigarette smoking and colorectal cancer incidence and mortality: systematic review and meta-analysis. Int 7 Cancer 2009; $124: 2406-15$

22. Dickinson J, Tsakonas E, Conner Gorber S, et al.; Canadian Task Force on Preventive Health Care. Recommendations on screening for cervical cancer. CMA7 2013;185:35-45.

23. Tonelli M, Conner Gorber S, Joffres M, et al.; Canadian Task Force on Pre- ventive Health Care. Recommendations on screening for breast cancer in average-risk women aged 40-74 years. CMA7 2011;183:1991-2001.

24. Hoffmeister M, Chang-Claude J, Brenner H. Validity of self-reported endoscopies of the large bowel and implications for estimates of colorectal cancer risk. Am 7 Epidemiol 2007;166:130-6.

25. Prevention. Vital signs: colorectal cancer screening test use - United States, 2012. MMWR Morb Mortal Wkly Rep 2013;62:881-8.

26. Spaeth A, Zwahlen M. Use of lower gastrointestinal endoscopy and fecal occult blood test in the 2007 Swiss Health Interview Survey respondents aged 50 years and older. Endoscopy 2013;45:560-6.

27. Stock C, Brenner H. Utilization of lower gastrointestinal endoscopy and fecal occult blood test in 11 European countries: evidence from the Survey of Health, Aging and Retirement in Europe (SHARE). Endoscopy 2010;42:546-56.

28. Shapiro JA, Seeff LC, Thompson TD, et al. Colorectal cancer test use from the 2005 National Health Interview Survey. Cancer Epidemiol Biomarkers Prev 2008; 17:1623-30

29. Shapiro JA, Klabunde CN, Thompson TD, et al. Patterns of colorectal cancer test use, including CT colonography, in the 2010 National Health Interview Survey. Cancer Epidemiol Biomarkers Prev 2012;21:895-904.

30. Torabi M, Green C, Nugent Z, et al. Geographical variation and factors associated with colorectal cancer mortality in a universal health care system. Chin 7 Gastroenterol Hepatol 2014;28:191-7.

31. Shaukat A, Mongin SJ, Geisser MS, et al. Long-term mortality after screening for colorectal cancer. N Engl 7 Med 2013;369:1106-14.

32. Scholefield JH, Moss SM, Mangham CM, et al. Nottingham trial of faecal occult blood testing for colorectal cancer: a 20-year follow-up. Gut 2012;61:1036-40.

33. Bernstein CN, Wajda A, Svenson LW, et al. The epidemiology of inflammatory bowel disease in Canada: a population-based study. Am 7 Gastroenterol 2006; 101:1559-68

Affiliations: IBD Clinical and Research Centre (Singh, Bernstein), University of Manitoba, Winnipeg, Man.; Internal Medicine (Singh, Bernstein), University of Manitoba, Winnipeg, Man.; Department of Medical Oncology and Hematology (Singh), CancerCare Manitoba, Winnipeg, Man.; Internal Medicine, Huntsman Cancer Institute (Samadder), University of Utah, Salt Lake City, Utah; Community Health Sciences (Ahmed), University of Manitoba, Winnipeg, Man.; Department of Epidemiology and Cancer Registry (Ahmed), CancerCare Manitoba, Winnipeg, Man.

Contributors: Harminder Singh and Rashid Ahmed were involved in the study concept and design. Rashid Ahmed obtained the data and performed the analysis. Harminder Singh drafted the initial draft of the manuscript. All of the authors were involved in the interpretation of data, critically revised the manuscript for important intellectual content, approved the version to be published and agreed to act as guarantors of the work.

Supplemental information: For reviewer comments and the original submission of this manuscript, please see www.cmajopen.ca/content/3/2/ E149/suppl/DCI. 